\title{
Andrographis paniculata transcriptome provides molecular insights into tissue-specific accumulation of medicinal diterpenes
}

\author{
Anchal Garg ${ }^{1}$, Lalit Agrawal ${ }^{2}$, Rajesh Chandra Misra', Shubha Sharma ${ }^{1}$ and Sumit Ghosh ${ }^{\text {* }}$
}

\begin{abstract}
Background: Kalmegh (Andrographis paniculata) has been widely exploited in traditional medicine for the treatment of infectious diseases and health disorders. Ent-labdane-related diterpene (ent-LRD) specialized (i.e., secondary) metabolites of kalmegh such as andrographolide, neoandrographolide and 14-deoxy-11,12-didehydroandrographolide, are known for variety of pharmacological activities. However, due to the lack of genomic and transcriptomic information, underlying molecular basis of ent-LRDs biosynthesis has remained largely unknown. To identify candidate genes of the ent-LRD biosynthetic pathway, we performed comparative transcriptome analysis using leaf and root tissues that differentially accumulate ent-LRDs.
\end{abstract}

Results: De novo assembly of Illumina HiSeq2000 platform-generated paired-end sequencing reads resulted into 69,011 leaf and 64,244 root transcripts which were assembled into a total of 84,628 unique transcripts. Annotation of these transcripts to the Uniprot, Kyoto Encyclopedia of Genes and Genomes (KEGG) and Carbohydrate-Active Enzymes (CAZy) databases identified candidate transcripts of the ent-LRD biosynthetic pathway. These included transcripts that encode enzymes of the plastidial 2C-methyl-D-erythritol-4-phosphate pathway which provides C5 isoprenoid precursors for the ent-LRDs biosynthesis, geranylgeranyl diphosphate synthase, class II diterpene synthase (diTPS), cytochrome P450 monooxygenase and glycosyltransferase. Three class II diTPSs (ApCPS1, ApCPS2 and ApCPS3) that showed distinct tissue-specific expression profiles and are phylogenetically related to the dicotyledon ent-copalyl diphosphate synthases, are identified. ApCPS1, ApCPS2 and ApCPS3 encode for 832-, 817- and 797- amino acids proteins of 55-63\% identity, respectively. Spatio-temporal patterns of transcripts and ent-LRDs accumulation are consistent with the involvement of APCPS1 in general (i.e., primary) metabolism for the biosynthesis of phytohormone gibberellin, ApCPS2 in leaf specialized ent-LRDs biosynthesis and ApCPS3 in root diterpene biosynthesis. Moreover, simple sequence repeats (SSRs) that might assist in genotyping and developing specific chemotypes were identified in transcripts of the specialized metabolic pathways, including ent-LRDs.

Conclusions: Comparative analysis of root and leaf transcriptomes disclosed novel genes of the ent-LRD biosynthetic pathway, including three class II diTPSs that showed discrete spatio-temporal expression patterns; thus, suggesting their participation into distinct diterpene metabolic pathways of kalmegh. Overall, these results will be useful in understanding molecular basis of the medicinal ent-LRDs biosynthesis and developing breeding strategies for improving their yields.

Keywords: Andrographis paniculata, ent-labdane-related diterpene, Medicinal compound, Specialized metabolism, Transcriptome

\footnotetext{
* Correspondence: sumitghosh@cimap.res.in

${ }^{1}$ Biotechnology Division, Council of Scientific and Industrial Research-Central Institute of Medicinal and Aromatic Plants, Lucknow 226015, India

Full list of author information is available at the end of the article
} 


\section{Background}

Kalmegh [Andrographis paniculata (Burm.f.) Wall. ex Nees], an annual herbaceous plant of the Acanthaceae family, is cultivated in Southern and Southeastern Asia for its diverse medicinal utilities $[1,2]$. During global flu epidemic in 1919, medicinal properties of kalmegh were effectively exploited to arrest spread of the contagious illness [3]. The genus Andrographis comprises of about 40 species, among these kalmegh is most popular as medicinal plant [4]. Although kalmegh has been widely used in traditional medicine in several Asian countries, Southern parts of India and Sri Lanka are considered as the centre of origin and diversity of the Andrographis species. In India, kalmegh is a predominant constituent in several ayurveda, unani, siddha and tribal medicine formulations for the treatment of infectious diseases and health disorders $[5,6]$. In vitro and in vivo bioactivity studies, using plant extracts as well as isolated compounds, revealed the utilities of kalmegh as hepatoprotective, anti-inflammatory, anticarcinogenic, anti-microbial, immunostimulatory, antioxidant and other healthpromoting activities [2, 7-9]. KalmCold ${ }^{\circ}$, a clinically tested phytochemical composition of kalmegh, has been proven to be effective for the treatment of upper respiratory tract infection [10].

Several bioactive specialized metabolites such as ent-labdane-related diterpenes (ent-LRDs), phenylpropanoids, flavonoids and xanthones were isolated from kalmegh $[11,12]$. However, ent-LRDs that accumulate in leaves such as andrographolide (AD), neoandrographolide (NAD) and 14-deoxy-11,12-didehydroandrographolide (DDAD) are considered as main bioactive constituents of kalmegh [7, 13-17] (Fig. 1). Among these ent-LRDs, andrographolide, the bitter principle of kalmegh, is most abundant and has been extensively studied for pharmacological activities such as immunostimulatory, anti-inflammatory and anticarcinogenic activities [18-22]. Andrographolide has been shown to inhibit proliferation of cancer cells by mitotic arrest and by activation of the intrinsic apoptotic pathway [21]. Andrographolide has also been shown to protect against cigarette smoke-induced oxidative lung injury via augmentation of the activities of anti-oxidative enzymes [8]. The anti-inflammatory activity of andrographolide was attributed to inhibition of the nuclear factor (NF)-kB pathway $[18,20]$. Moreover, andrographolide was suggested to be effective in reducing chronic stress-triggered pathologies by regulating corticosterone and cytokine homeostasis [23].

The low yield of bioactive principles in kalmegh has led to increased market rate. Although, good-quality dried leaves of kalmegh could be sold for US $\$ 5 / \mathrm{kg}$, purified compounds and their derivatives may cost as much as US $\$ 100,000 / \mathrm{kg}$ from specialist chemical suppliers [7]. Understanding the molecular basis of specialized metabolite biosynthetic pathways and their regulation shall be effective in increasing yield as well as designing specific chemotypes following biotechnological and molecular breeding approaches $[24,25]$. However, the genes/enzymes involved in the biosynthesis of medicinally active specialized metabolites of kalmegh are yet to be identified and functionally characterized. The lack of transcriptomic and genomic resources is a hindrance for understanding the specialized metabolite biosynthetic pathways of kalmegh. Only 41 Expressed Sequence Tags (ESTs) and 60 nucleotide sequences of kalmegh are available in the National Center for Biotechnology Information (NCBI) GenBank database. In recent times, high throughput transcriptome sequencing using Illumina short-read sequencing platform has become a powerful approach to develop reference transcriptome for gene identification in non-model plants [26-31].

In the present study, to generate a reference transcriptome of kalmegh for the identification of genes of the specialized metabolic pathways, transcriptome sequencing and de novo assembly have been performed using tissues that either accumulate high level of ent-LRDs (leaf) or do not accumulate ent-LRDs (root). Comparative analysis of leaf and root transcriptomes revealed candidate genes for the biosynthesis of ent-LRDs. Three class II diterpene synthases (diTPSs) are identified. These class II diTPSs showed discrete spatio-temporal expression patterns; suggesting their participation into distinct diterpene metabolic pathways of kalmegh.

\section{Methods}

\section{Plant materials and growth conditions}

Kalmegh (Cv. CIM-Megha) seeds were collected from the National Gene Bank for Medicinal and Aromatic Plants (CSIR-CIMAP). Seeds were germinated in presterilized soil and, at the second true leaf stage, seedlings were transplanted into earthen pots $(15 \mathrm{~cm}$ height and internal diameter) with a mixture (2:1) of soil and vermicompost. Plants were grown in a glass house during the months of July-October at $26-28{ }^{\circ} \mathrm{C}$ under the natural light. Plants were watered daily with application of Hoagland's solution once a week. Roots, stems and matured green leaves were obtained from two-month-old plants. Samples were also collected at germinating seeds (GS) and seedlings at cotyledonary leaf stage (CLS). All samples were washed with $\mathrm{RO}$ water, frozen immediately in liquid nitrogen and stored at $-80{ }^{\circ} \mathrm{C}$.

\section{RNA isolation and CDNA library preparation}

Total RNA was isolated using TRIzol reagent according to the manufacturer's protocol (Invitrogen). cDNA library preparation was performed according to the Illumina TruSeq RNA library protocol outlined in "TruSeq RNA Sample Preparation Guide" (Part \# 15008136; Rev. 


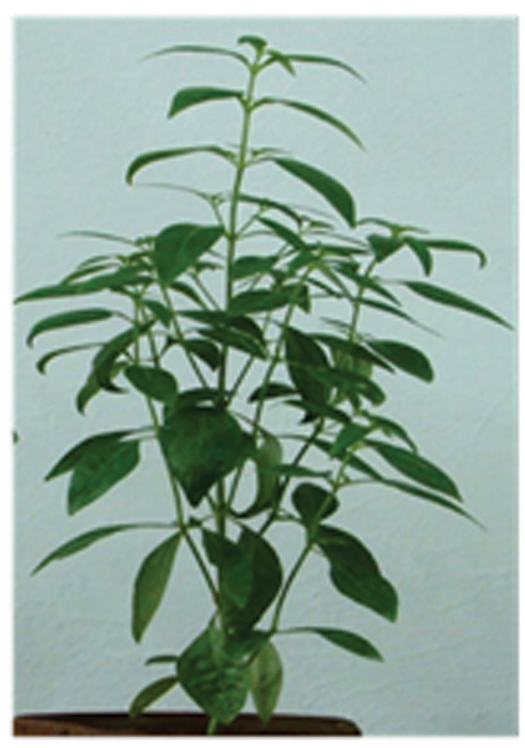

Vegetative phase

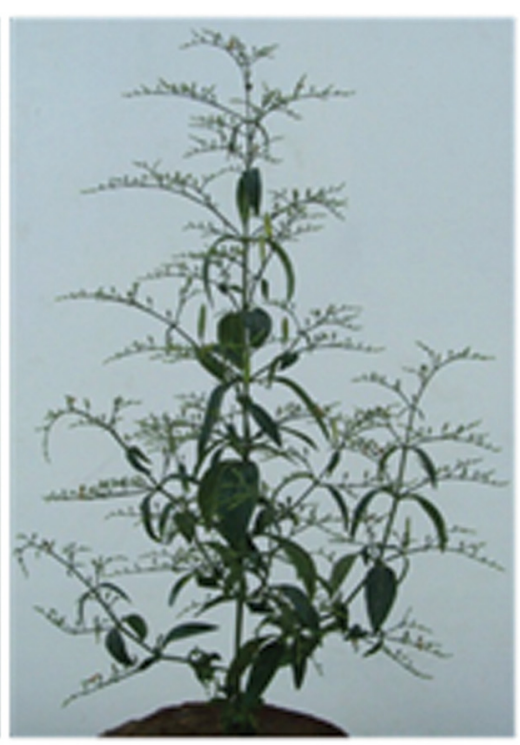

Reproductive phase<smiles>[3H][C@]12CCC(=C)[C@H](C/C=C3/C(=O)OC[C@@H]3O)[C@@]1(C)CC[C@H](O)[C@]2(C)CO</smiles>

$\mathrm{AD}$<smiles>C=C1CCC[C@]2(C)[C@H](CCC3=CCOC3=O)[C@](C)(COC(C)=O)[C@@H]2CC1</smiles>

NAD<smiles>C=C1CC[C@@]2(CO)C[C@H](O)[C@@](C)(CC[C@@H]2C)[C@@H]1/C=C/C1=CCOC1=O</smiles>

DDAD

Fig. 1 The medicinal plant kalmegh and major ent-LRDs that accumulate in leaves. AD, andrographolide; NAD, neoandrographolide; DDAD, 14-deoxy-11,12-didehydroandrographolide

A; Nov 2010). In brief, mRNAs were isolated from $1.0 \mu \mathrm{g}$ total RNA following Poly-A RNA purification method. Further, purified mRNAs were fragmented with divalent cations at $94{ }^{\circ} \mathrm{C}$ for $4 \mathrm{~min}$ and reversetranscribed with Superscript III Reverse transcriptase by using random hexamers. Second strand cDNAs were produced in the presence of DNA Polymerase I and $\mathrm{RNaseH}$, and cDNAs were cleaned up using Agencourt Ampure XP SPRI beads (Beckman Coulter). Following end repair and addition of $\mathrm{A}$ base, Illumina Adapters were ligated to the cDNAs and SPRI cleanup was performed. Amplification of the cDNA library was carried out following eight cycles of PCR for the enrichment of adapter-ligated fragments. The cDNA library was quantified using Nanodrop and validated for quality by running an aliquot on High Sensitivity Bioanalyzer Chip (Agilent). The libraries showed peak spread over a range of 250-700 bp with the effective sequencing insert size of 130-580 bp, excluding adaptor sequences.

\section{Transcriptome sequencing, raw data processing and de novo assembly}

Paired-end (100 bp) sequencing of root and leaf cDNA libraries was carried out using Illumina HiSeq2000 platform. Sequence reads of the leaf and root libraries are deposited in the NCBIs Short Read Archive database under the accession number SRP044357. Raw reads were quality checked using SeqQC V2.2 tool (Genotypic Technology Pvt. Ltd.) to remove adapters and low quality bases. De novo assembly of processed reads was 
performed using Velvet-1.2.10 [32] for various hash length (k-mers). K-mer of 55 was found to be better than others considering various parameters like total number of contigs generated, maximum contig length, total contig length and less number of N's. Further, Oases 0.2.08 [33] was performed using Velvet-1.2.10 assembly, for the generation of final transcripts for root and leaf tissues. These transcripts were clustered using CD-HIT [34] at $95 \%$ sequence identity to generate a non-redundant reference transcriptome for kalmegh. The strategy for the transcriptome assembly is presented in Additional file 1: Figure S1.

\section{Transcriptome annotation and differential gene expression analysis}

Transcripts were similarity searched (ncbi-BLAST-2.2.29) against the sequences of Acanthaceae family deposited at the Uniprot-viridiplantae database. In addition, annotation was also carried out with rice and Arabidopsis proteins available at the Uniprot database. Gene Ontology was predicted for the annotated transcripts using GO information from the Uniprot database. Metabolic pathway analysis was carried out using KAAS Server [35] following default parameters and using Arabidopsis and rice as the model organisms. Transcripts encoding predicted glycosyltransferase enzymes were identified following annotation against eukaryotic glycosyltransferase sequences collected from the Carbohydrate-Active Enzymes (CAZy) database [36]. Cytochrome P450 monoxygenase families were identified based on the sequence similarity to the cytochrome P450 monoxygenase sequences available at the Uniprot database. For the identification of transcription factor families, transcripts were annotated to the Plant Transcription Factor Database (http://plntfdb.bio.uni-potsdam.de/v3.0). For the digital gene expression analysis (DGE), processed reads were aligned to the assembled transcriptome after generating unigenes to obtain read counts using custom perl scripts (Genotypic Technology Pvt. Ltd.). Reads were first aligned using "Bowtie tool" [37] and "Awk scripting" was used to generate the read count profile from the output file (.sam) of Bowtie alignment. Differential gene expression analysis was carried out using DESeq software [38] considering root sample as control, as explained with a flow diagram in Additional file 1: Figure S2.

\section{Simple sequence repeats (SSRs) prediction}

SSR prediction was carried out using MISA perl script (http://pgrc.ipk-gatersleben.de/misa/download/misa.pl).

Transcripts were checked for mono-repeats occurring at least ten times, di-repeats occurring at least six times and tri/tetra/penta/hexa-repeats occurring at least five times within a sequence. SSR was classified as complex when two SSRs were identified within $100 \mathrm{bp}$ distance of each other.

\section{Quantitative real time PCR}

RNAs were isolated, treated with DNase I and purified using RNeasy Mini Kit (Qiagen) as described previously [39]. Three independent isolations consisting of at least three plants for each group were performed. RNA quality was monitored by analysing the A260/280 ratio and resolving on $1.2 \%(w / v)$ agarose gel. Four microgram of total RNAs were reverse-transcribed using superscript II (Invitrogen) and processed for qRT-PCR analysis using 7900 HT Fast Real Time PCR (Applied Biosystems) as described previously [39]. Oligonucleotide primers used in qRT-PCR amplification are listed in Additional file 2: Table S1. Relative gene expression was determined based on the $2^{-\Delta \Delta C t}$ method using actin (KJ494921) as endogenous control.

\section{High performance liquid chromatography}

Fresh tissues from germinating seeds (GS), seedlings at cotyledonary leaf stage (CLS), roots, stems and leaves were harvested and quickly frozen in liquid nitrogen. Using liquid nitrogen, and with the help of a pestle and mortar, frozen tissue was ground into fine powder. Ground tissue $(500 \mathrm{mg}$ ) was extracted twice with $5 \mathrm{ml}$ of methanol, evaporated to dryness and finally dissolved into $2 \mathrm{ml}$ of methanol. HPLC analysis was carried out using a HPLC-UV (Shimadzu LC-10A, Tokyo, Japan) system as described previously [40]. Stock solution (1 $\mathrm{mg} \mathrm{ml}^{-1}$ ) of authentic standard andrographolide (Sigma) was prepared in methanol and used for standard curve preparation.

\section{Results and discussion}

Transcriptome sequencing, de novo assembly and quality assessment

For organisms that lack reference genome, high throughput transcriptome sequencing using Illumina short-read sequencing platform combined with de novo transcriptome assembly has become a standard method in generating reference transcriptome with in-depth coverage [26-31]. Therefore, to identify candidate genes involved in the biosynthesis of medicinally active ent-LRDs, a reference transcriptome of kalmegh was generated following high-throughput sequencing of transcriptome using Illumina HiSeq2000 platform. As described in Materials and Methods, complementary DNA (cDNA) libraries were prepared from leaf and root tissues that accumulate contrasting levels of ent-LRDs [41] (Fig. 8b). Paired-end (100 bp) sequencing yielded 101.78 million and 78.48 million of raw reads for leaf and root, respectively. After processing of raw reads, 96.14 million and 74.85 million of high quality reads representing $9322.83 \mathrm{Mb}$ and $7280.98 \mathrm{Mb}$ of high quality bases were obtained for leaf and root, respectively. These high quality reads were utilized for de novo transcriptome assembly following 
Velvet_1.2.10 and Oases_0.2.08 software packages [32, 33]. The overall strategy of transcriptome assembly and analysis is presented in Additional file 1: Figure S1. By combining Velvet-1.2.10 and Oases_0.2.08 analyses, a total of 69,011 contigs with N50 of $926 \mathrm{bp}$ and average length of $667 \mathrm{bp}$ were generated for leaf transcriptome (Table 1). However, in case of root, a total of 64,244 contigs with N50 of $992 \mathrm{bp}$ and average length of $692.5 \mathrm{bp}$ were generated. The minimum length of leaf and root contigs was $200 \mathrm{bp}$. A large number of contigs $(\sim 55 \%)$ were in the size range of 200-500 bp (Fig. 2a). On the other hand, $\sim 25 \%$ and $\sim 15 \%$ of contigs were in the size range of 501-1000 bp and 1001-3000 bp, respectively. These sequence data were in accordance to the transcriptomes reported for other plant species using Illumina shortread sequencing platform [27-30].

In order to evaluate quality of the assembled transcripts, identity and coverage of the sequences with the Uniprot protein database were determined following BlastX analysis (Additional file 3: Table S2 and Additional file 4: Table S3). The translation products of $87.25 \%$ of the leaf and $86.80 \%$ of the root annotated transcripts showed $\geq 50 \%$ sequence identity with protein sequences deposited to the Uniprot database. Moreover, $\geq 70 \%$ sequence coverage was recorded for $25.91 \%$ (average length-1559.12 bp) of the leaf and $28.64 \%$ (average length-1557.04 bp) of the root annotated transcripts, representing nearly full-length transcripts. Besides, $\geq 50 \%$ sequence coverage was noticed for $39.06 \%$ (average length-1360.97 bp) and $41.85 \%$ (average length-1373.27 bp) of the leaf and root annotated transcripts, respectively. The average lengths of the transcripts with $<50 \%$ coverage were 508.64 and $516.22 \mathrm{bp}$ for leaf and root, respectively. These parameters reflected the quality of the assembled transcripts.

Table 1 Statistics for Illumina HiSeq2000 sequencing and de novo transcriptome assembly

\begin{tabular}{lll}
\hline Description & Leaf & Root \\
\hline Total number of HQ reads & $96,143,552$ & $74,854,072$ \\
Total number of bases (Mb) & 9563.22 & 7454.82 \\
HQ bases (\%) & 97.48 & 97.66 \\
Reads with non-ATGC characters (\%) & 0.32 & 0.33 \\
GC content (\%) & 47.94 & 46.84 \\
Reads assembled (\%) & 81.15 & 81.71 \\
Total number of contigs & 69,011 & 64,244 \\
Average length (bp) & 667 & 692.5 \\
Total length (bp) & $46,032,575$ & $44,489,873$ \\
N50 (bp) & 926 & 992 \\
\hline
\end{tabular}

\section{Annotation of the leaf and root transcriptomes}

To assign putative function, transcripts were searched (BLAST version 2.2.29+) for homology to the annotated sequences in the Uniprot database, rice, Arabidopsis and Acanthaceae family (Fig. 2b; Additional file 3: Table S2 and Additional file 4: Table S3) and also processed for the Gene Ontology (GO) classifications (Additional file 1: Figure S3) and Kyoto Encyclopedia of Genes and Genomes (KEGG) pathway analysis (Additional file 5: Table S4 and Additional file 6: Table S5). Functional annotation was assigned for $\sim 60 \%$ of the transcripts based on similarity to the annotated sequences in the Uniprot database. However, $\sim 30 \%$ of the transcripts were annotated to the rice and Arabidopsis sequences. Because of limited transcript information on Acanthaceae family species, only $\sim 4 \%$ of the transcripts showed similarity with the annotated sequences of the Acanthaceae family species. BlastX search to the Uniprot database also provided information on to the species distribution of the annotated transcripts. When transcripts with $\geq 75 \%$ of sequence identity to the annotated sequences in the Uniprot database were classified based on top-hit species distribution, Genlisea aurea represented top-hit species with similarity to 2446 leaf and 2114 root transcripts (Fig. 2c). This was in accordance with the fact that both G. aurea and $A$. paniculata belong to the order Lamiales. Only few transcripts showed similarity to the Acanthaceae family species such as A. paniculata and Avicennia marina. This reflected the lack of transcripts sequences of the Acanthaceae family species in the database.

KEGG pathways were annotated to 4044 leaf and 3965 root transcripts (Additional file 5: Table S4 and Additional file 6: Table S5). In addition to several general (i.e., primary) metabolic pathways, transcriptome also represented various specialized (i.e., secondary) metabolic pathways such as terpenoids and phenylpropanoids (Fig. 3). Specialized metabolites of these classes were previously isolated from kalmegh, however, their biosynthetic pathway genes were not identified [11, 12, 42]. Functional analysis of the candidate genes of the specialized metabolic pathways shall be useful to understand the molecular and biochemical basis for the accumulation of medicinally active specialized metabolites in kalmegh.

\section{Combined assembly of leaf and root transcriptomes and identification of the differentially expressed transcripts}

To generate a non-redundant reference transcriptome of kalmegh, leaf and root assembled transcripts were clustered using CD-HIT at $95 \%$ sequence identity [34]. By following this approach, a total of 84,628 transcripts with an average length of 688.53 bp were generated (Table 2). For the identification of differential transcripts, these assembled unique transcripts $(84,628)$ were considered as master control transcript and the read count 

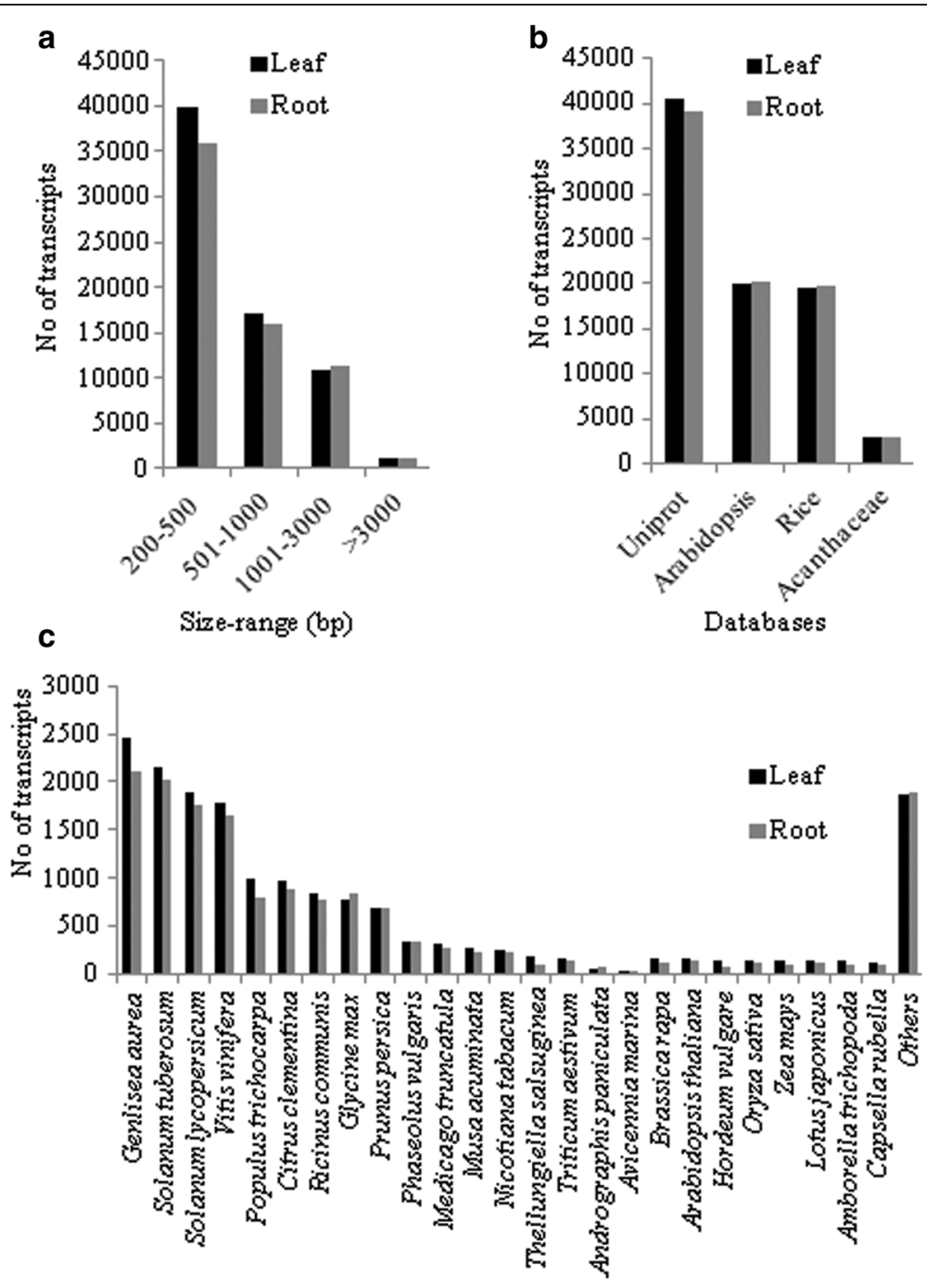

Fig. 2 Size distribution and annotation of assembled transcripts. a Size distribution of transcripts. b Annotation of transcripts to different sequence databases was carried out on the basis of sequence similarity as determined by BLAST (version 2.2.29+) analysis. c Top-hit species distribution of transcripts showing $\geq 75 \%$ sequence identity with annotated sequences of the Uniprot database

profiles were first determined for the leaf and root Illumina reads using Bowtie tool [37] and further proceed for the differential gene expression analysis using DegSeq tool [38] (Additional file 1: Figure S2). This digital gene expression (DGE) analysis revealed 6277 and 5418 transcripts with expression only in leaf and root tissues, respectively. However, 19,564 and 16,678 transcripts were up-regulated and down-regulated, respectively, in leaf as compared to root. To authenticate DGE-based expression profiles of the transcripts, a correlation with quantitative RT-PCR (qRT-PCR)-based expression patterns was determined. For this, 35 transcripts that are related to the specialized metabolism were selected (Fig. 4). The overall correlation coefficient $(r)$ of $0.9398\left(r^{2}=0.8832\right)$ indicated a very high level of correlation between DGE- and qRT-PCR-based expression profiles of the transcripts. Therefore, DGE- based expression profiles may be considered to identify transcripts involved in tissue-specific accumulation of specialized metabolites in kalmegh. To identify candidate transcripts of the ent-LRD biosynthetic pathway, transcripts were classified according to the DGE-based expression patterns in leaf and root tissues. DGE-based expression profiles of the transcripts with annotation to the diterpene biosynthetic pathway and to different CYP450, GT and transcription factor families are presented in Table 3; Additional file 1: Figure S4, Additional file 7: Table S6, Additional file 8: Table S7 and Additional file 9: Table S8.

\section{Transcripts of the diterpene biosynthetic pathway}

In plants, plastidial 2C-methyl-D-erythritol-4-phosphate (MEP) and cytosolic mevalonic acid (MEV) pathways 


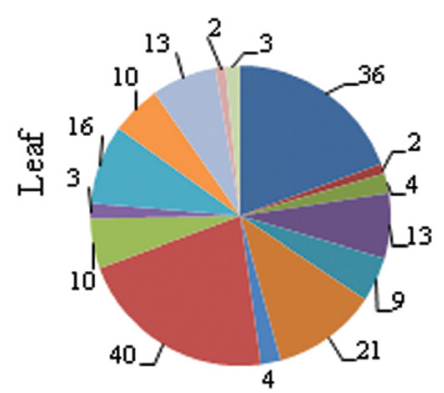

Terpenoid backbomebiosynthesis

andole alkaloid biosynthes is

- Monoterpencid biosynthesis

Limonene and pinene degradation

Diterpenoidbiosynthesis

-Carotenoidbiosynthesis

-Ses quiterperoid and triterpenoid biosynthes is

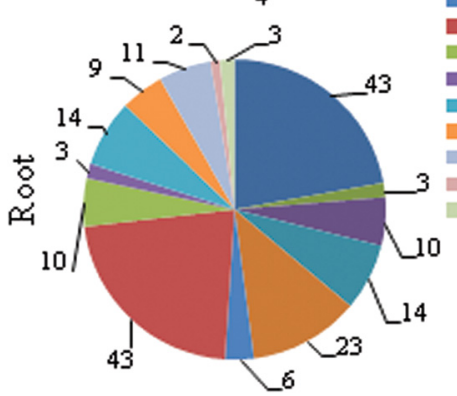

-Phenylpropanoid biosynthesis

Flavonoid biosynthes is.

- Flavone and flavool biosynthesis

Stitbenoid, diarylheptanoid and gingerolb iosynthesis

Is oquinoline alkaloid biosynthesis

Tropane, piperidine and pyidine alkaloid biosynthesis

Ghucosinolateb iosynthes is

Caffeine metabolism

Fig. 3 Annotation of transcripts to different specialized metabolic pathways based on the KEGG database

provide two $5 \mathrm{C}$ isoprenoid building blocks, dimethylallyl diphosphate (DMAPP) and isopentenyl diphosphate (IPP), for the biosynthesis of diverse terpene metabolites [43]. IPP and DMAPP derived from the MEP pathway are converted to monoterpenes, diterpenes, and tetraterpenes, whereas those derived from the MEV pathway are converted to sesquiterpenes and triterpenes. However, cross-talk between these two pathways in biosynthesis of some terpenes was also recognised [44-46]. Previously, a major role of the MEP pathway and a minor role of the MEV pathway for supplying the $5 \mathrm{C}$ isoprenoid precursors for the biosynthesis of andrographolide were reported [47]. Transcripts predicted to encode all the enzymes of the MEP and MEV pathways are identified in kalmegh transcriptome (Table 3; Fig. 5); further demonstrating the quality and in-depth coverage of the transcriptome database generated in this study. Interestingly, four transcripts for DXS, three for DXR and two each for HDS, HDR, AACT, HMGS, HMGR,

Table 2 Combined assembly of leaf and root transcriptomes

\begin{tabular}{ll}
\hline Description & No. \\
\hline Total number of contigs & 8,4628 \\
Average length (bp) & 688.53 \\
Total length (bp) & $5,826,9163$ \\
Contigs (200-500 bp) & 47,592 \\
Contigs (501-1000 bp) & 21,164 \\
Contigs (1001-3000 bp) & 14,528 \\
Contigs (>3000 bp) & 1344 \\
N50 (bp) & 976 \\
\hline
\end{tabular}

PMK, MVD and IDI were revealed in kalmegh transcriptome. This observation suggests the likely existence of multiple isomers for these enzymes in kalmegh.

The second stage of diterpene biosynthesis involves head-to-tail condensation of three IPP and one DMAPP to a C20 compound geranylgeranyl diphosphate (GGPP) (Fig. 5). This prenyltransfer reaction is catalyzed by the plastidial geranylgeranyl diphosphate synthase (GGPS). In ent-LRD biosynthetic pathway, GGPP is further cyclized into ent-diterpenyl diphosphate e.g., ent-copalyl diphosphate (ent-CPP) following protonation-initiated cyclization mechanism catalyzed by the class II diterpene synthase (diTPS). Ent-diterpenyl diphosphate then acts as substrate for the class I diTPS that catalyzes further cyclization and/or rearrangement reactions [48, 49]. Thus, based on structures of kalmegh ent-LRDs, the involvement of class I and class II diTPSs, CYP450s and GTs enzymes in the biosynthesis of ent-LRDs was hypothesized (Fig. 5). From kalmegh transcriptome database, three partial transcripts for the GGPS and three full-length transcripts for class II diTPSs with homology to the ent-copalyl diphosphate synthase (ent-CPS) are identified (Table 3). Besides, several transcripts for CYP450s and GTs are also recognized (Fig. 5; Additional file 7: Table S6 and Additional file 8: Table S7). Transcripts that encode MEP pathway enzymes, GGPS, class II diTPS, CYP450 and GT, and preferentially expressed in leaf tissue (Fig. 6; Table 3; Additional file 7: Table S6, Additional file 8: Table S7 and Additional file 1: Figure S4) are potential candidates for the biosynthesis of entLRDs in kalmegh. Although, two class I diTPSs with sequence similarity with ent-kaurene synthase are 


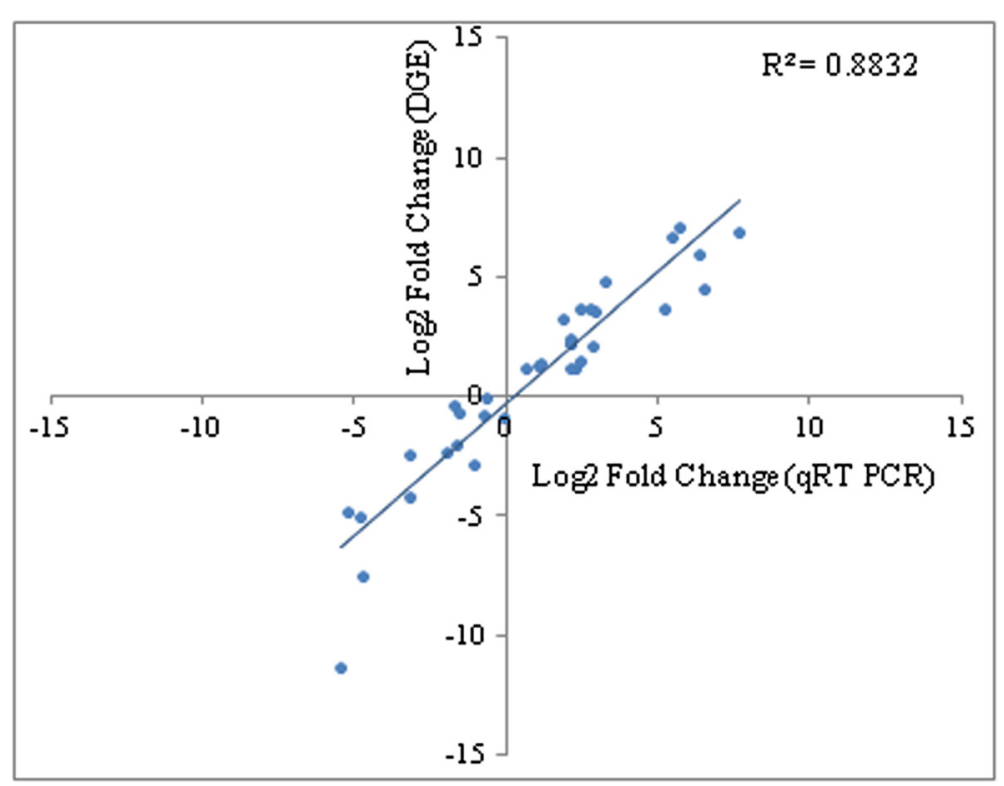

Fig. 4 Correlation of DGE- and qRT-PCR-based expression profiles of transcripts. Expression profiles of 35 transcripts that are related to specialized metabolism were selected for the analysis. Data are presented as log2FoldChange (leaf vs root). GRT - PCR data represents average of three biological replicates. Differential transcript expression determined by qRT - PCR in root and leaf tissues was statistically evaluated either at $P<0.05$ or $P<0.01$

identified (Table 3), none of them preferentially expressed in leaf. Thus, their involvement in the biosynthesis of entLRD medicinal compounds in kalmegh may be excluded.

In contrast to diTPSs, not many CYP450s and GTs of specialized diterpene biosynthetic pathways are known. Some of the characterized members include CYP450s of taxol, phytoalexins and diterpene resin acid, and GTs of steviol glycoside biosynthetic pathways [50-56]. The majority of CYP450s of the specialized terpene metabolism belong to the CYP71 and CYP85 clans [57, 58]. From kalmegh transcriptome database, 147 transcripts that belong to the clan 71 (CYP families 71, 76, 78, 81-84, 93, 98, 706, 736) and clan 85 (CYP families 85, 90, 707, 716) are identified (Additional file 7: Table S6 and Additional file 1: Figure S4). Among these, 45 transcripts preferentially expressed in leaf compared to root. On the other hand, family 1 GTs (GT1) are the key players in glycosylation of specialized metabolites $[59,60]$. Among the total of 161 GT1s of kalmegh, 55 GT1s preferentially expressed in leaf compared to root (Additional file 8: Table S7 and Additional file 1: Figure S4). Further studies on these leaf-expressed CYP450s of the clan 71 and clan 85 , and GT1s can lead to the identification of potential oxidase(s) and GT(s) of kalmegh ent-LRD biosynthetic pathway.

\section{Identification of simple sequence repeats in diterpene biosynthetic pathway transcripts}

Simple sequence repeats (SSRs) are often considered most efficient and reliable molecular markers for detecting genetic variations in plants [61]. Therefore, to identify functional SSRs of kalmegh, leaf and root transcripts were examined for the presence of microsatellite motifs using MIcroSAtellite (MISA) tool (http://pgrc.ipk-gatersleben.de/misa). A total of 16,485 potential SSRs were identified in 13,805 leaf transcripts (Additional file 10: Table S9). Whereas, 15,911 SSRs were detected in 13,213 root transcripts. Moreover, 2194 leaf and 2200 root transcripts were detected with more than one SSRs. Di-nucleotide repeats were the most abundant SSRs in leaf and root transcripts with 5194 and 5023 SSRs, respectively. The numbers of compound SSRs were 1877 and 1895 in leaf and root transcripts, respectively. The complete lists of SSRs detected in leaf and root transcripts are provided in Additional file 11: Table S10 and Additional file 12: Table S11. Interestingly, several SSRs were also identified in transcripts of the specialized metabolic pathways, including terpenes and phenylpropanoids (Additional file 13: Table S12). SSRs were detected for the transcripts of the MEP pathway enzymes (DXS, MDS and HDR), GGPS and class II diTPSs (ApCPS2, ApCPS3). These SSRs could be useful in genotyping cultivars and developing specific chemotypes of kalmegh following marker-assisted selection.

\section{Identification and analysis of diterpene synthases}

Annotation of the kalmegh transcriptome revealed three diTPSs that showed close phylogenetic relationship with the dicotyledons monofunctional class II diTPSs of entCPP product specificity (Fig. 7). These are ApCPS1 
Table 3 List of transcripts related to diterpene biosynthesis

\begin{tabular}{|c|c|c|c|c|}
\hline Pathway & Transcript ID & Transcript annotation & Transcript length (bp) & Expression $^{\mathrm{a}}$ \\
\hline \multirow[t]{14}{*}{ MEP } & ApU56176 & 1-Deoxy-D-xylulose-5-phosphate synthase (DXS) & 2421 & 1.210593385 \\
\hline & ApU12883 & 1-Deoxy-D-xylulose-5-phosphate synthase (DXS) & 1284 & -0.620028298 \\
\hline & ApU13057 & 1-Deoxy-D-xylulose-5-phosphate synthase (DXS) & 2647 & -0.064469923 \\
\hline & ApU57524 & 1-Deoxy-D-xylulose-5-phosphate synthase (DXS) & 2529 & 4.485204599 \\
\hline & ApU8165 & 1-Deoxy-D-xylulose-5-phosphate reductoisomerase (DXR) & 1019 & 2.090903695 \\
\hline & ApU50057 & 1-Deoxy-D-xylulose-5-phosphate reductoisomerase (DXR) & 1084 & 2.274895363 \\
\hline & Ap2567 & 1-Deoxy-D-xylulose-5-phosphate reductoisomerase (DXR) & 1013 & 4.853346 \\
\hline & ApU70472 & 2-C-Methyl-D-erythritol 4-phosphate cytidylyltransferase (MCT) & 1277 & 3.71794589 \\
\hline & ApU7163 & 4-Diphosphocytidyl-2-C-methyl-D-erythritol kinase (CMK) & 1553 & 1.228243799 \\
\hline & ApU3039 & 2-C-Methyl-D-erythritol 2,4-cyclodiphosphate synthase (MDS) & 611 & 3.330961011 \\
\hline & ApU45802 & (E)-4-Hydroxy-3-methylbut-2-enyl-diphosphate synthase (HDS) & 2631 & 3.613822951 \\
\hline & ApU45495 & (E)-4-Hydroxy-3-methylbut-2-enyl-diphosphate synthase (HDS) & 2664 & -0.839568655 \\
\hline & ApU67412 & 4-Hydroxy-3-methylbut-2-enyl diphosphate reductase (HDR) & 1830 & 5.990336076 \\
\hline & ApU393 & 4-Hydroxy-3-methylbut-2-enyl diphosphate reductase (HDR) & 1773 & 3.743023121 \\
\hline \multirow[t]{2}{*}{ MEP/MEV } & ApU9344 & Isopentenyl-diphosphate delta-isomerase (IDI) & 1165 & -2.855299841 \\
\hline & ApU80862 & Isopentenyl-diphosphate delta-isomerase (IDI) & 426 & Root only \\
\hline \multirow[t]{11}{*}{ MEV } & ApU45787 & Acetyl-CoA C-acetyltransferase (AACT) & 758 & 0.073827478 \\
\hline & ApU3388 & Acetyl-CoA C-acetyltransferase (AACT) & 1132 & -2.201783665 \\
\hline & ApU29957 & Hydroxymethylglutaryl-CoA synthase (HMGS) & 1009 & -0.970530757 \\
\hline & ApU46957 & Hydroxymethylglutaryl-CoA synthase (HMGS) & 1127 & -0.971997692 \\
\hline & ApU2925 & Hydroxymethylglutaryl-CoA reductase (HMGR) & 2235 & 1.932136157 \\
\hline & ApU46382 & Hydroxymethylglutaryl-CoA reductase (HMGR) & 2454 & -2.018649251 \\
\hline & ApU51503 & Mevalonate kinase (MK) & 1202 & -0.741169194 \\
\hline & ApU4232 & Phosphomevalonate kinase (PMK) & 1439 & -1.11100184 \\
\hline & ApU58988 & Phosphomevalonate kinase (PMK) & 1171 & -0.311783537 \\
\hline & ApU51812 & Diphosphomevalonate decarboxylase (MVD) & 573 & -1.349116106 \\
\hline & ApU9903 & Diphosphomevalonate decarboxylase (MVD) & 974 & -1.562474564 \\
\hline \multirow[t]{17}{*}{ Diterpene } & ApU8378 & Geranylgeranyl diphosphate synthase (GGPS) & 1749 & 1.018074228 \\
\hline & ApU952 & Geranylgeranyl diphosphate synthase (GGPS) & 984 & 2.233068085 \\
\hline & ApU55421 & Geranylgeranyl diphosphate synthase (GGPS) & 1522 & 1.364502354 \\
\hline & ApU53774 & Ent-copalyl diphosphate synthase (Ent-CPS) & 2623 & Root only \\
\hline & ApU55291 & Ent-copalyl diphosphate synthase (Ent-CPS) & 2567 & -0.80001242 \\
\hline & ApU48901 & Ent-copalyl diphosphate synthase (Ent-CPS) & 2654 & 7.119944191 \\
\hline & ApU14593 & Ent-kaurene synthase (KS) & 1544 & -1.728307905 \\
\hline & ApU66227 & Ent-kaurene synthase (KS) & 1707 & -2.414805387 \\
\hline & ApU14229 & Ent-kaurene oxidase (KO) & 1966 & -3.699209001 \\
\hline & ApU51425 & Ent-kaurenoic acid hydroxylase (KAO) & 1938 & -6.870577872 \\
\hline & ApU51353 & Ent-kaurenoic acid hydroxylase (KAO) & 1911 & -3.81097751 \\
\hline & ApU77665 & Gibberellin 2-oxidase (GA2ox) & 666 & -4.234871361 \\
\hline & ApU79135 & Gibberellin 2-oxidase (GA2ox) & 628 & -3.741831349 \\
\hline & ApU23389 & Gibberellin 2-oxidase (GA2ox) & 1330 & 4.108980573 \\
\hline & ApU10203 & Gibberellin 20-oxidase (GA20ox) & 635 & 3.315288446 \\
\hline & ApU51228 & Gibberellin 20-oxidase (GA20ox) & 1395 & 2.941320399 \\
\hline & ApU45906 & Gibberellin 3-beta-dioxygenase (GA3ox) & 1089 & -5.116226864 \\
\hline
\end{tabular}


Table 3 List of transcripts related to diterpene biosynthesis (Continued)

\begin{tabular}{llll}
\hline ApU57038 & Momilactone-A synthase (MAS) & 1003 & -8.215762538 \\
ApU58121 & Momilactone-A synthase (MAS) & 846 & -9.795908022 \\
ApU67465 & Momilactone-A synthase (MAS) & 972 & Root only \\
ApU1116 & Momilactone-A synthase (MAS) & 1145 & 2.550321912 \\
\hline
\end{tabular}

alog2FoldChange (leaf vs root) based on DGE

(ApU55291), ApCPS2 (ApU48901) and ApCPS3 (ApU53774) (Table 3). Similar to class II diTPSs, the highly conserved DXDD motif that is essential for the protonation-initiated cyclization of GGPP was identified in ApCPS1, ApCPS2 and ApCPS3, following multiple sequence alignment (Additional file 1: Figure S5). Sequence analysis revealed that ApCPS1, ApCPS2 and
ApCPS3 encode for 832-, 817- and 797- amino acids proteins with calculated molecular masses of 95.45, 93.43 and $90.81 \mathrm{kD}$, respectively. At the amino acid sequence level, ApCPS1 shared 55.2 and $57.21 \%$ identities with ApCPS2 and ApCPS3, respectively (ClustalW score, http://www.ebi.ac.uk/). However, ApCPS2 shared $63.36 \%$ amino acid identity with ApCPS3. Like other plant

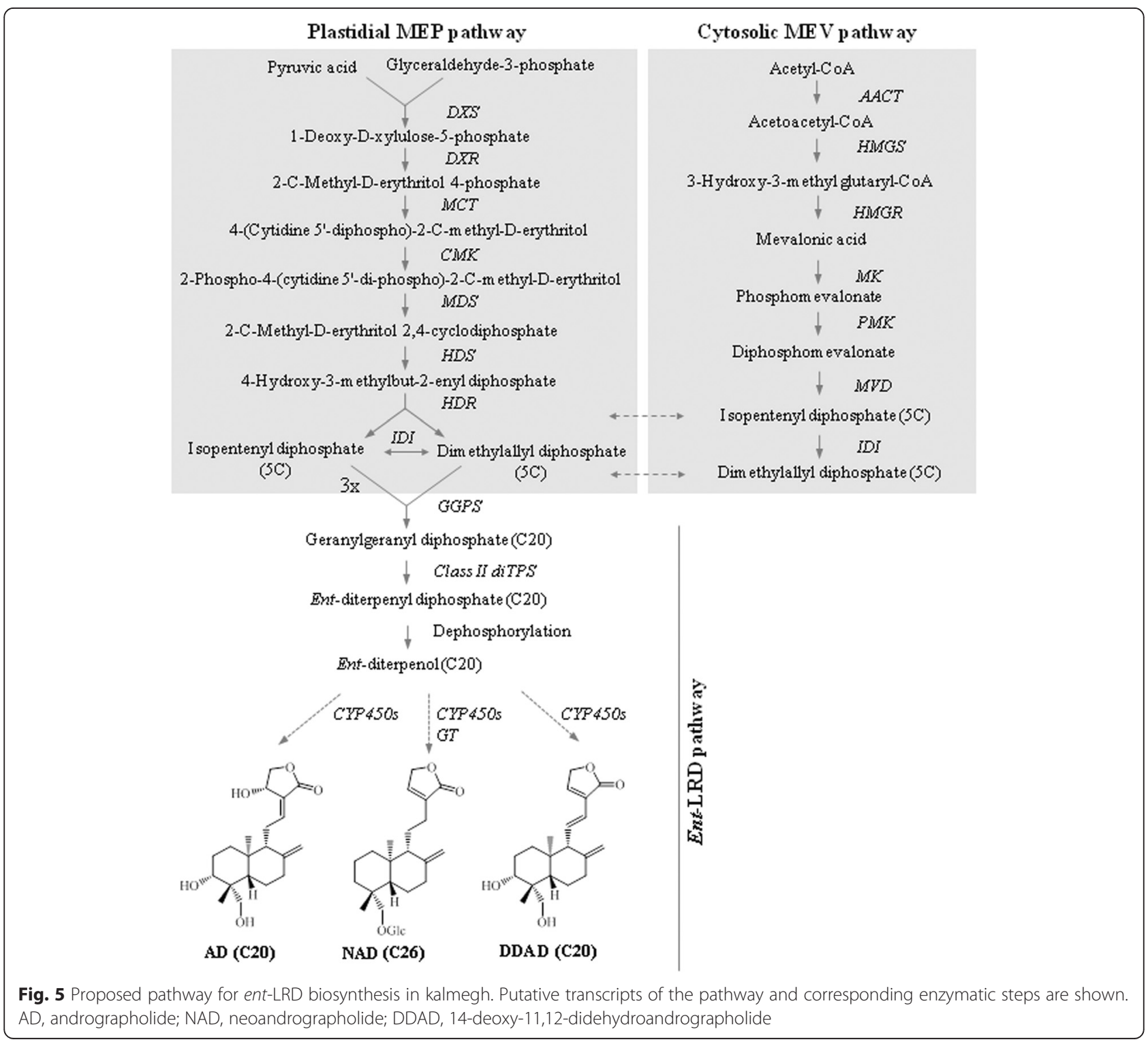




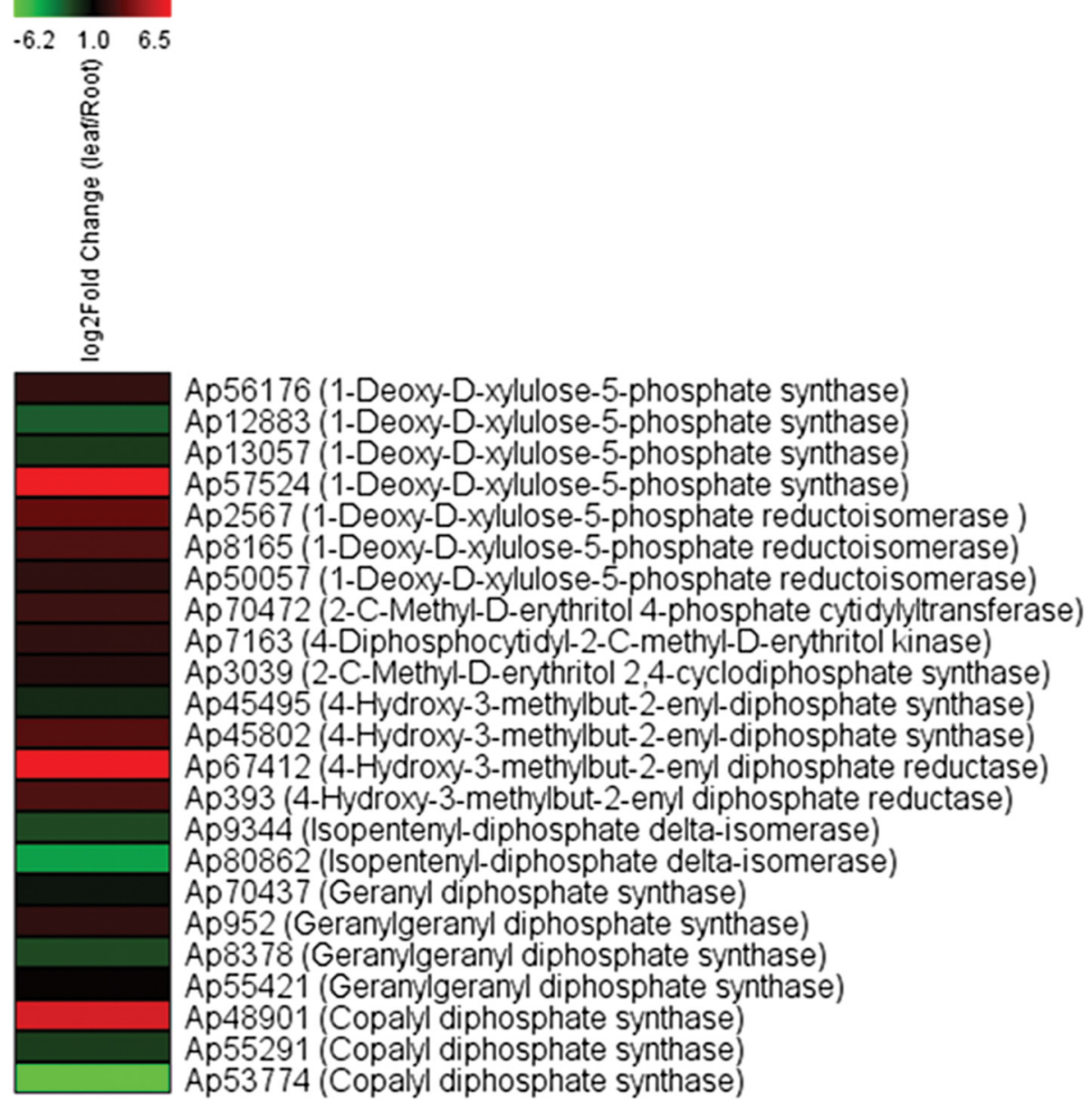

Fig. 6 Relative expression level of transcripts, putatively related to ent-LRD biosynthesis. Expression profiles of the MEP pathway transcripts, GGPSs and class II diTPSs were determined through qRT - PCR in root and leaf tissues. Data are presented as log2FoldChange (leaf vs root) obtained from three biological replicates. Differential transcript expression in root and leaf tissues was statistically evaluated either at $P<0.05$ or $P<0.01$

diTPSs, N-terminal transit peptides for the chloroplast localization were recognised in ApCPS1, ApCPS2 and $A p C P S 3$, following iPSORT (http://ipsort.hgc.jp/) and Predotar v. 1.30. (http://urgi.versailles.inra.fr/predotar/predotar.html) analysis.

ApCPS1, ApCPS2 and ApCPS3 exhibited dissimilar expression patterns in leaf and root tissues (Fig. 6). The transcripts levels of ApCPS1 were comparable in leaf and root tissues. However, ApCPS2 showed high level of transcript accumulation in leaf and low level of transcript accumulation in root. In contrast, ApCPS3 transcripts were detected at very high level in root and at very low level in leaf. This divergent expression pattern of ApCPS1, ApCPS2 and ApCPS3 indicated their role in different diterpene metabolic pathways of kalmegh, although, their involvement in same biosynthetic pathway with functional redundancy cannot be completely excluded. In order to determine potential functions of ApCPS1, ApCPS2 and ApCPS3 in kalmegh, transcripts levels were analysed in different plant organs and during seedling development stages following qRT - PCR (Fig. 8a). Moreover, to correlate transcript expression with metabolite accumulation pattern, the level of andrographolide, the most abundant ent-LRD of kalmegh, was determined in plant organs and during seedling developmental ages following HPLC analysis (Fig. 8b and Additional file 1: Figure S6). Maximum transcript level for ApCPS1 was detected in stem (4.03fold), followed by seedlings at cotyledonary leaf stage (CLS, 2.79-fold) as compared to germinating seeds (GS). ApCPS1 transcript was also detected during seed germination. Because ent-CPP also serves as precursor for the biosynthesis of phytohormone gibberellin (GA) that is known to promote seed germination, seedling development and stem elongation in plant species [48, 49, 62, 63], we suggest the role of $A p C P S 1$ in general metabolism by providing ent-LRD precursor for GA biosynthesis. In contrast to ApCPS1, ApCPS2 transcript expression was 


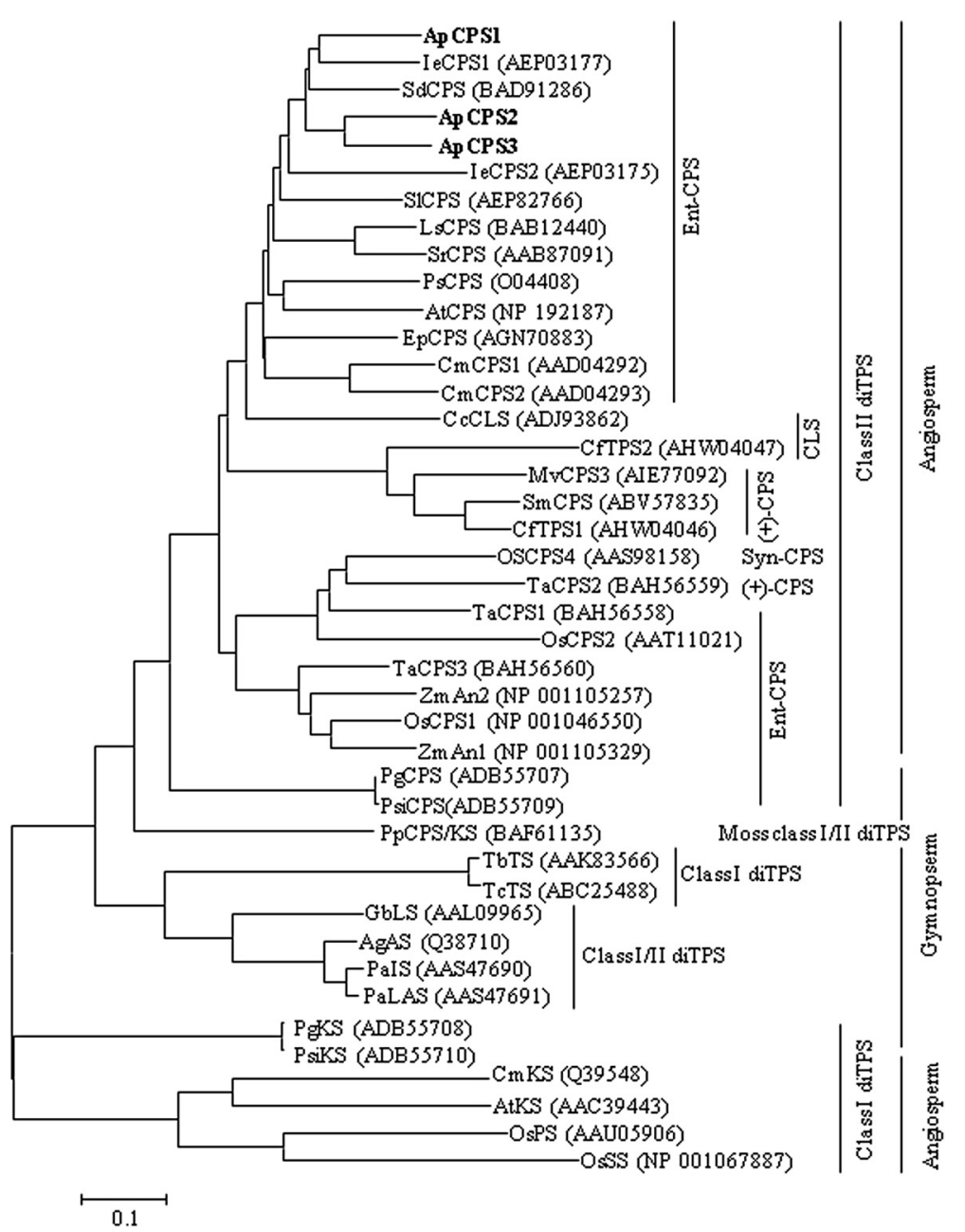

Fig. 7 The phylogenetic relationship of kalmegh class II diTPSs with diTPSs of angiosperms, gymnosperms and moss. The evolutionary history was inferred using the Neighbor-Joining method. The evolutionary distances were computed using the Poisson correction method and are in the units of the number of amino acid substitutions per site. Evolutionary analyses were conducted in MEGA6. Ent-CPS, syn-CPS, (+)-CPS denote ent-, syn- and (+)/normal copalyl diphosphate synthase, respectively. CLS means copal-8-ol diphosphate synthase

maximum in leaf (104.34-fold), followed by stem (14.19fold) as compared to GS. However, very low level of ApCPS2 transcript was detected during seed germination and in seedlings at the CLS stage, as compared to leaf and stem. Based on the transcript expression and ent-LRD metabolite accumulation patterns in plant organs and during seedling developmental ages, the role of ApCPS2 in tissuespecific accumulation of medicinal ent-LRDs was anticipated (Fig. 8a and b). Although, bioactive ent-LRDs accumulate at high level in leaf (86.84-fold compared to GS), they were undetected or detected at very low level in root [41] (Fig. 8b). The high level expression of ApCPS3 in kalmegh root (1491.41-fold as compared to GS) suggests biosynthesis of $\operatorname{LRD}(\mathrm{s})$ in root which is/are yet to be identified. The role of ApCPS3 in root diterpene phytoalexin biosynthesis cannot be excluded. We hypothesized this function of ApCPS3 because class II diTPSs are known to play role in root phytoalexin biosynthesis in plants [64-66]. Moreover, kalmegh transcripts putatively encoding momilactone-A synthase, a phytoalexin biosynthetic pathway enzyme [67], also expressed at high level in roots (Table 3).

In medicinal ent-LRDs biosynthetic pathway of kalmegh, ent-diterpenyl diphosphate/ent-CPP produced from class II diTPS activity might acts as substrate of class I diTPS for further hydrolysis of the phosphate group without additional cyclization and rearrangement steps. This class I diTPS activity may be related to the class I diTPS activity of the bifunctional class I/II diTPS of Selaginella moellendorffii [68]. However, transcripts that show sequence similarity to the class I diTPSs and preferentially express in leaf tissue which accumulates medicinal ent-LRDs, could not be identified. Although two transcripts for class I diTPSs with sequence 

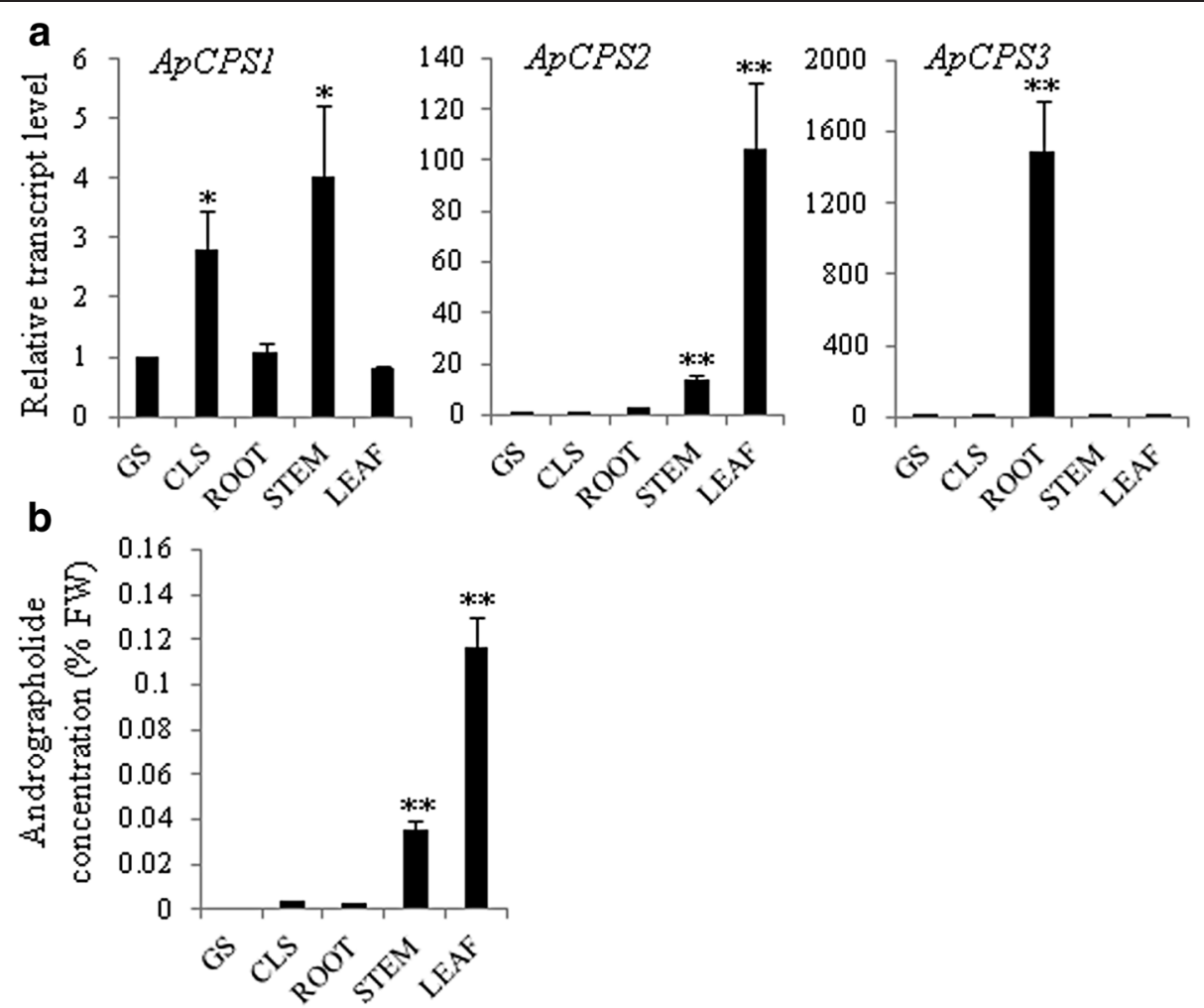

Fig. 8 Tissue-specific expression profiles of class II diTPSs and accumulation pattern of major ent-LRD andrographolide. a Transcript levels were determined by qRT-PCR analysis. Data are presented as the mean ( \pm SE) of three biological replicates. $\mathbf{b}$ Andrographolide levels in different tissues were determined by high-performance liquid chromatography analysis. Data are presented as the mean ( \pm SE) of three biological replicates. Andrographolide was either undetected or detected at very low level in GS, CLS and root samples. ${ }^{*} P<0.05$ and ${ }^{* *} P<0.01$ compared with GS sample

homology to the ent-kaurene synthase are recognised (Table 3), both of them preferentially expressed in root compared to leaf and have a stretch of overlapping sequences; suggesting that the two contigs might in fact represent a single transcript. Therefore, the role of an endogenous phosphatase for the conversion of ent-diterpenyl diphosphate/ent-CPP into ent-diterpenol/ent-copalol is also likely, as was shown for the biosynthesis of diterpenol in Nicotiana [69, 70]. Additional structural diversities in ent-LRDs including oxygen functionality and glycosylation might be brought about by the activities of the CYP450s and GTs, respectively, that preferentially express in leaf tissue (Additional file 7: Table S6, Additional file 8: Table S7 and Additional file 1: Figure S4).

\section{Conclusion}

Several bioactive specialized metabolites, including entLRDs were isolated from kalmegh [11, 12]; however, their biosynthesis was not studied. The present study was undertaken with the aim to identify candidate genes involved in the biosynthesis of specialized metabolites with special emphasis on ent-LRDs that are considered as the major medicinally active components of kalmegh. Independent sequencing of leaf and root transcriptomes using Illumina
HiSeq2000 platform and individual as well as combined assembly of the transcriptomes resulted in generation of a reference transcriptome of kalmegh with in-depth coverage. This experimental approach also helped us to gather information regarding expression patterns of the identified transcripts in ent-LRD accumulating and non-accumulating tissues. Transcripts predicted to encode all the enzymes of the MEP and MEV pathways, GGPSs, diTPSs, CYP450s and GTs are identified and classified, and based on transcript expression patterns, their role in the tissue-specific accumulation of medicinal ent-LRDs is discussed. Our results indicate the occurrence of three isoforms for the class II diTPS (ApCPS1, ApCPS2 and ApCPS3) in kalmegh. These genes showed discrete spatio-temporal expression patterns suggesting their participation into distinct diterpene metabolic pathways of kalmegh. Data suggest the role of ApCPS1 in general metabolism (GA biosynthesis); while $A p C P S 2$ is potentially involved in the biosynthesis of medicinal ent-LRDs in leaf. In contrast, expression pattern of ApCPS3 suggests its involvement in the biosynthesis of root diterpenes, possibly phytoalexins. In addition, SSRs were identified in the transcripts of the specialized metabolic pathways, including ent-LRDs. These SSRs might be useful in selecting and developing desired chemotypes of kalmegh 
following molecular breeding approaches. Taken together, these results will help us to understand the molecular and regulatory basis of tissue-specific accumulation patterns of medicinally active specialized metabolites in kalmegh and to develop molecular breeding strategies to improve their yields.

\section{Additional files}

Additional file 1: Figure S1. Flow chart displaying strategies employed for de novo transcriptome analysis. Figure S2. Flow chart displaying strategies employed for digital gene expression analysis. Figure S3. Gene ontology classification of root and leaf transcripts. Figure S4. Transcription factor, cytochrome P450 monoxygenase and glycosyltransferase families. Figure S5. Amino acid sequence comparison of class II diTPSs of kalmegh. Figure S6. HPLC chromatograms of metabolites extracted from anderographolide-accumulating (Leaf and stem) and non-accumulating (root, GS, CLS) tissues of A. paniculata. (PDF $367 \mathrm{~kb}$ )

Additional file 2: Table S1. List of the primers used in this study. (DOCX $20 \mathrm{~kb}$ )

Additional file 3: Table S2. Annotation of leaf transcripts to the Uniprot database. (XLS $17060 \mathrm{~kb}$ )

Additional file 4: Table S3. Annotation of root transcripts to the Uniprot database. (XLS $16520 \mathrm{~kb}$ )

Additional file 5: Table S4. KEGG pathways represented by leaf transcripts. (XLS $719 \mathrm{~kb}$ )

Additional file 6: Table S5. KEGG pathways represented by root transcripts. (XLS $710 \mathrm{~kb}$ )

Additional file 7: Table S6. Putative cytochrome P450

monooxygenases. (XLSX $47 \mathrm{~kb}$ )

Additional file 8: Table S7. Putative Glycosyltransferases. (XLS 195 kb)

Additional file 9: Table S8. Putative transcription factors. (XLSX $45 \mathrm{~kb}$ )

Additional file 10: Table S9. A summary of SSRs identified in leaf and root transcriptomes. (DOCX $11 \mathrm{~kb}$ )

Additional file 11: Table S10. SSRs in leaf transcripts. (XLS $2660 \mathrm{~kb}$ )

Additional file 12: Table S11. SSRs in root transcripts. (XLS $1818 \mathrm{~kb}$ )

Additional file 13: Table S12. SSRs in specialized metabolic pathway transcripts. (XLSX $13 \mathrm{~kb}$ )

\section{Competing interests}

The authors declare that they have no competing interests.

\section{Authors' contributions}

AG and RCM performed experiments; AG, LA, RCM, SS and SG analysed data; SG designed the study and wrote the manuscript. All authors read and approved the final manuscript.

\section{Acknowledgments}

This work was supported by a research grant (SR/FT/LS-36/2012) to S.G. from the Science and Engineering Research Board, Department of Science and Technology, Government of India. The authors gratefully acknowledge the Council of Scientific and Industrial Research-Central Institute of Medicinal and Aromatic Plants for providing research facilities; Dr. Alok Lehri, the Council of Scientific and Industrial Research-National Botanical Research Institute for help in HPLC analysis and Genotypic Technology (P) Ltd (Bangalore, India) for transcriptome sequencing and de novo assembly. AG and RCM acknowledge the Department of Science and Technology and the Council of Scientific and Industrial Research, respectively, for pre-doctoral fellowships.

\section{Author details}

${ }^{1}$ Biotechnology Division, Council of Scientific and Industrial Research-Central Institute of Medicinal and Aromatic Plants, Lucknow 226015, India. ${ }^{2}$ Council of Scientific and Industrial Research-National Botanical Research Institute, Lucknow 226001, India.
Received: 4 February 2015 Accepted: 18 August 2015

Published online: 02 September 2015

\section{References}

1. Chopra RN, Nayer SL, Chopra IC. Glossary of Indian medicinal plants. New Delhi, India: Council of Scientific and Industrial Research; 1956.

2. Akbar S. Andrographis paniculata, a review of pharmacological activities and clinical effects. Altern Med Rev. 2011;16:66-77.

3. Sharma SN, Sinha RK, Sharma DK, Jha Z. Assessment of intra-specific variability at morphological, molecular and biochemical level of Andrographis paniculata (Kalmegh). Curr Sci. 2009;96:402-8.

4. Boopathi CA. Andrographis spp:: a source of bitter compounds for medicinal use. Anc Sci Life. 2000;19:164-8.

5. Khare CP. Indian medicinal plant: an illustrated dictionary. Berlin, Germany: Springer; 2007. doi:10.1007/978-0-387-70638-2.

6. Raina AP, Gupta V, Sivaraj N, Dutta M. Andrographis paniculata (Burm. f.) Wall. ex Nees (kalmegh), a traditional hepatoprotective drug from India. Genet Resour Crop Evol. 2013;60:1181-9.

7. Valdiani A, Kadir MA, Tan SG, Talei D, Abdullah MP, Nikzad S. Nain-e Havandi Andrographis paniculata present yesterday, absent today, a plenary review on underutilized herb of Iran's pharmaceutical plants. Mol Biol Rep. 2012;39:5409-24.

8. Guan SP, Tee W, Ng DS, Chan TK, Peh HY, Ho WE, et al. Andrographolide protects against cigarette smoke-induced oxidative lung injury via augmentation of Nrf2 activity. Br J Pharmacol. 2013;168:1707-18.

9. Bardi DA, Halabi MF, Hassandarvish P, Rouhollahi E, Paydar M, Moghadamtousi SZ, et al. Andrographis paniculata leaf extract prevents thioacetamide-induced liver cirrhosis in rats. PLoS ONE. 2014;9:e109424. doi:10.1371/journal.pone.0109424.

10. Saxena RC, Singh R, Kumar P. A randomized double blind placebo controlled clinical evaluation of extract of Andrographis paniculata (KalmCold) in patients with uncomplicated upper respiratory tract infection. Phytomedicine. 2010;17(3-4):17178-185.

11. Koteswara RY, Vimalamma G, Rao CV, Tzeng Y. Flavonoids and andrographolides from Andrographis paniculata. Phytochemistry. 2004;65:2317-21.

12. Li W, Xu X, Zhang H, Ma C, Fong $H$, van Breemen R, et al. Secondary metabolites from Andrographis paniculata. Chem Pharm Bull. 2007;55:455-8.

13. Ooi JP, Kuroyanagi M, Sulaiman SF, Muhammad TS, Tan ML. Andrographolide and 14-deoxy-11, 12-didehydroandrographolide inhibit cytochrome P450s in HepG2 hepatoma cells. Life Sci. 2011;88:447-54.

14. Mondal S, Roy P, Das S, Halder A, Mukherjee A, Bera T. In vitro susceptibilities of wild and drug resistant leishmania donovani amastigote stages to andrographolide nanoparticle: role of vitamin E derivative TPGS for nanoparticle efficacy. PLoS ONE. 2013;8:e81492. doi:10.1371/ journal.pone.0081492. eCollection 2013.

15. Lin HH, Shi MD, Tseng HC, Chen JH. Andrographolide sensitizes the cytotoxicity of human colorectal carcinoma cells toward cisplatin via enhancing apoptosis pathways in vitro and in vivo. Toxicol Sci. 2014;139:108-20.

16. Raghavan R, Cheriyamundath S, Madassery J. 14-Deoxy-11,12didehydroandrographolide inhibits proliferation and induces GSH-dependent cell death of human promonocytic leukemic cells. J Nat Med. 2014;68:387-94.

17. Wang J, Tan XF, Nguyen VS. A quantitative chemical proteomics approach to profile the specific cellular targets of andrographolide, a promising anticancer agent that suppresses tumor metastasis. Mol Cell Proteomics. 2014:13:876-86.

18. Xia YF, Ye BQ, Li YD. Andrographolide attenuates inflammation by inhibition of NF-kappa B activation through covalent modification of reduced cysteine 62 of p50. J Immunol. 2004;173:4207-17.

19. Abu-Ghefreh $\mathrm{AA}$, Canatan $\mathrm{H}$, Ezeamuzie $\mathrm{Cl}$. In vitro and in vivo anti-inflammatory effects of andrographolide. Int Immunopharmacol. 2009;9:313-8.

20. Bao Z, Guan S, Cheng C. A novel antiinflammatory role for andrographolide in asthma via inhibition of the nuclear factor-kB pathway. Am J Respir Crit Care Med. 2009;179:657-65.

21. Zhang ZR, Al Zaharna M, Wong MMK, Chiu SK, Cheung HY. Taxifolin enhances andrographolide-induced mitotic arrest and apoptosis in human prostate cancer cells via spindle assembly checkpoint activation. PLOS ONE. 2013;8:e54577. doi:10.1371/journal.pone.0054577.

22. Chen YY, Hsieh CY, Jayakumar T, Lin KH, Chou DS, Lu WJ, et al. Andrographolide induces vascular smooth muscle cell apoptosis through a 
SHP-1-PP2A-p38MAPK-p53 cascade. Sci Rep. 2014;4:5651. doi:10.1038/ srep05651.

23. Thakur AK, Soni UK, Rai G, Chatterjee SS, Kumar V. Protective effects of Andrographis paniculata extract and pure andrographolide against chronic stress-triggered pathologies in rats. Cell Mol Neurobiol. 2014;34:1111-21.

24. Lange BM, Ahkami A. Metabolic engineering of plant monoterpenes, sesquiterpenes and diterpenes-current status and future opportunities. Plant Biotech J. 2013;11:169-96.

25. Paddon CJ, Westfall PJ, Pitera DJ, Benjamin K, Fisher K, McPhee D, et al. High-level semi-synthetic production of the potent antimalarial artemisinin. Nature. 2013;496:528-32.

26. Wang Z, Gerstein M, Snyder M. RNA-Seq: a revolutionary tool for transcriptomics. Nat Rev Genet. 2009;10:57-63.

27. He M, Wang Y, Hua W, Zhang Y, Wang Z. De Novo sequencing of hypericum perforatum transcriptome to identify potential genes involved in the biosynthesis of active metabolites. PLOS ONE. 2012;7:e42081. doi:10.1371/journal.pone.0042081.

28. Huang J, Lu X, Yan H, Chen S, Zhang W, Huang R, et al. Transcriptome characterization and sequencing-based identification of salt-responsive genes in Millettia pinnata, a semi-mangrove plant. DNA Res. 2012;19:195207

29. Verma P, Shah N, Bhatia S. Development of an expressed gene catalogue and molecular markers from the de novo assembly of short sequence reads of the lentil (Lens culinaris Medik.) transcriptome. Plant Biotechnol J. 2013;11:894-905

30. Ranjan A, Ichihashi Y, Farhi M, Zumstein K, Townsley B, David-Schwartz R, et al. De novo assembly and characterization of the transcriptome of the parasitic weed Cuscuta pentagona identifies genes associated with plant parasitism. Plant Physiol. 2014;166:1186-99.

31. Upadhyay S, Phukan UJ, Mishra S, Shukla RK. De novo leaf and root transcriptome analysis identified novel genes involved in steroidal sapogenin biosynthesis in Asparagus racemosus. BMC Genomics. 2014;15:746. doi:10.1186/1471-2164-15-746.

32. Zerbino DR, Birney E. Velvet: algorithms for de novo short read assembly using de Bruijn graphs. Genome Res. 2008;18:821-9. doi:10.1101/gr.074492.107.

33. Schulz MH, Zerbino DR, Vingron M, Birney E. Oases: robust de novo RNAseq assembly across the dynamic range of expression levels. Bioinformatics. 2012;28:1086-92. doi:10.1093/bioinformatics/bts094.

34. Fu L, Niu B, Zhu Z, Wu S, Li W. CD-HIT: accelerated for clustering the nextgeneration sequencing data. Bioinformatics. 2012;28:3150-2.

35. Moriya Y, Itoh M, Okuda S, Yoshizawa A, Kanehisa M. KAAS: an automatic genome annotation and pathway reconstruction server. Nucleic Acids Res. 2007;35:W182-5.

36. Lombard V, Golaconda Ramulu H, Drula E, Coutinho PM, Henrissat B. The carbohydrate-active enzymes database (CAZy) in 2013. Nucleic Acids Res. 2014:42:D490-5.

37. Langmead B, Trapnell C, Pop M, Salzberg SL. Ultrafast and memory-efficient alignment of short DNA sequences to the human genome. Genome Biol. 2009;10:R25. doi:10.1186/gb-2009-10-3-r25.

38. Anders $\mathrm{S}$, Huber W. Differential expression analysis for sequence count data. Genome Biol. 2010;11:R106. doi:10.1186/gb-2010-11-10-r106.

39. Misra RC, Maiti P, Chanotiya CS, Shanker K, Ghosh S. Methyl jasmonateelicited transcriptional responses and pentacyclic triterpene biosynthesis in sweet basil. Plant Physiol. 2014;164:1028-44.

40. Tewari SK, Niranjan A, Lehri A. Variations in yield, quality, and antioxidant potential of kalmegh (Andrographis paniculata Nees) with soil alkalinity and season. J Herbs Spices Med Plants. 2010;16:41-50.

41. Pholphana N, Rangkadilok N, Saehun J, Ritruechai S, Satayavivad J. Changes in the contents of four active diterpenoids at different growth stages in Andrographis paniculata (Burm.f.) Nees (Chuanxinlian). Chin Med. 2013;8:2. doi:10.1186/1749-8546-8-2.

42. Matsuda T, Kuroyanagi M, Sugiyama S, Umehara K, Ueno A, Nishi K. Cell differentiation-inducing diterpenes from Andrographis paniculata Nees. Chem Pharm Bull (Tokyo). 1994;42:1216-25.

43. Tholl D, Lee S. Terpene specialized metabolism in Arabidopsis thaliana. Arabidopsis Book. 2011;9:e0143. doi:10.1199/tab.0143

44. De-Eknamkul W, Potduang B. Biosynthesis of beta-sitosterol and stigmasterol in Croton sublyratus proceeds via a mixed origin of isoprene units. Phytochemistry. 2003;62:389-98
45. Paetzold H, Garms S, Bartram S, Wieczorek J, Uros-Gracia EM, RodriguezConcepcion $\mathrm{M}$, et al. The isogene 1-deoxy-D-xylulose 5-phosphate synthase 2 controls isoprenoid profiles, precursor pathway allocation, and density of tomato trichomes. Mol Plant. 2010;3:904-16.

46. Schramek N, Wang H, Romisch-Margl W, Keil B, Radykewicz T, Winzenhorlein $\mathrm{B}$, et al. Artemisinin biosynthesis in growing plants of Artemisia annua A 13 CO2 study. Phytochemistry. 2010;71:179-87.

47. Srivastava N, Akhila A. Biosynthesis of andrographolide in Andrographis paniculata. Phytochemistry. 2010;71:1298-304.

48. Cyr A, Wilderman PR, Determan M, Peters RJ. A modular approach for facile biosynthesis of labdane-related diterpenes. J Am Chem Soc. 2007;129:6684-5.

49. Peters RJ. Two rings in them all, the labdane-related diterpenoids. Nat Prod Rep. 2010;27:1521-30.

50. Jennewein S, Long RM, Williams RM, Croteau R. Cytochrome p450 taxadiene 5alpha-hydroxylase, a mechanistically unusual monooxygenase catalyzing the first oxygenation step of taxol biosynthesis. Chem Biol. 2004;11:379-87.

51. Hamberger B, Ohnishi T, Hamberger B, Seguin A, Bohlmann J. Evolution of diterpene metabolism: Sitka spruce CYP720B4 catalyzes multiple oxidations in resin acid biosynthesis of conifer defense against insects. Plant Physiol. 2011;157:1677-95.

52. Wu Y, Hillwig ML, Wang Q, Peters RJ. Parsing a multifunctional biosynthetic gene cluster from rice: iochemical characterization of CYP71Z6 \& 7. FEBS Lett. 2011;585:3446-51.

53. Wang Q, Hillwig ML, Peters RJ. CYP99A3: functional identification of a diterpene oxidase from the momilactone biosynthetic gene cluster in rice. Plant J. 2011;65:87-95.

54. Wang Q, Hillwig ML, Wu Y, Peters RJ. CYP701A8: a rice entkaurene oxidase paralog diverted to more specialized diterpenoid metabolism. Plant Physiol. 2012;158:1418-25.

55. Richman A, Swanson A, Humphrey T. Functional genomics uncovers three glucosyltransferases involved in the synthesis of the major sweet glucosides of Stevia rebaudiana. Plant J. 2005:41:56-67.

56. Madhav H, Bhasker S, Chinnamma M. Functional and structural variation of uridine diphosphate glycosyltransferase (UGT) gene of Stevia rebaudianaUGTSr involved in the synthesis of rebaudioside A. Plant Physiol Biochem. 2013;63:245-53.

57. Nelson D, Werck-Reichhart D. A P450-centric view of plant evolution. Plant J. 2011;66:194-211.

58. Zerbe P, Hamberger B, Yuen MM, Chiang A, Sandhu HK, Madilao LL, et al. Gene discovery of modular diterpene metabolism in nonmodel systems. Plant Physiol. 2013;162:1073-91.

59. Simon C, Langlois-Meurinne M, Didierlaurent L, Chaouch S, Bellvert $F$, Massoud $\mathrm{K}$, et al. The secondary metabolism glycosyltransferases UGT73B3 and UGT73B5 are components of redox status in resistance of Arabidopsis to Pseudomonas syringae pv. tomato. Plant Cell Environ. 2014;37:1114-29.

60. Gachon CM, Langlois-Meurinne M, Saindrenan P. Plant secondary metabolism glycosyltransferases: the emerging functional analysis. Trends Plant Sci. 2005:10:542-9.

61. Choudhary S, Gaur R, Gupta S. EST-derived genic molecular markers: development and utilization for generating an advanced transcript map of chickpea. Theor Appl Genet. 2012;124:1449-62.

62. Hedden P, Kamiya Y. Gibberellin biosynthesis, enzymes, genes and their regulation. Annu Rev Plant Physiol Plant Mol Biol. 1997;48:431-60.

63. Hartweck LM. Gibberellin signalling. Planta. 2008;229:1-13.

64. Otomo K, Kenmoku H, Oikawa H, Konig WA, Toshima H, Mitsuhashi W, et al. Biological functions of ent- and syn-copalyl diphosphate synthases in rice, key enzymes for the branch point of gibberellin and phytoalexin biosynthesis. Plant J. 2004;39:886-93.

65. Prisic S, Xu M, Wilderman PR, Peters RJ. Rice contains two disparate entcopalyl diphosphate synthases with distinct metabolic functions. Plant Physiol. 2004;136:4228-36.

66. Wu Y, Zhou K, Toyomasu T, Sugawara C, Oku M, Abe S, et al. Functional characterization of wheat copalyl diphosphate synthases sheds light on the early evolution of labdane-related diterpenoid metabolism in the cereals. Phytochemistry. 2012;84:40-6.

67. Shimura K, Okada A, Okada K, Jikumaru Y, Ko KW, Toyomasu T, et al. Identification of a biosynthetic gene cluster in rice for momilactones. J Biol Chem. 2007;282:34013-8. 
68. Mafu S, Hillwig ML, Peters RJ. A novel labda-7,13e-dien-15-ol-producing bifunctional diterpene synthase from Selaginella moellendorffii. ChemBioChem. 2011;12:1984-7.

69. Sallaud C, Giacalone C, Topfer R, Goepfert S, Bakaher N, Rosti S, et al. Characterization of two genes for the biosynthesis of the labdane diterpene Z-abienol in tobacco (Nicotiana tabacum) glandular trichomes. Plant J. 2012;72:1-17.

70. Zerbe P, Chiang A, Dullat H, O'Neil-Johnson M, Starks C, Hamberger B, et al. Diterpene synthases of the biosynthetic system of medicinally active diterpenoids in Marrubium vulgare. Plant J. 2014;79:914-27.

Submit your next manuscript to BioMed Central and take full advantage of:

- Convenient online submission

- Thorough peer review

- No space constraints or color figure charges

- Immediate publication on acceptance

- Inclusion in PubMed, CAS, Scopus and Google Scholar

- Research which is freely available for redistribution 